\title{
Aging Effects on Neuromuscular Activity in Karate Practitioners
}

António Manuel Vitória Vences de Brito ${ }^{1,2,3}$, Mário André Ferreira Rodrigues ${ }^{1,2}$, Wojciech Jan Cynarski ${ }^{3,4}$, Carlos Gutiérrez García ${ }^{3,5}$

1. Sport Sciences School of Rio Maior, Polytechnic Institute of Santarém, 2040-413 Rio Maior, Portugal

2. Life Quality Research Centre-CIEQV, Complexo Andaluz, Apartado 279, 2001-904 Santarém, Portugal

3. International Martial Arts and Combat Sports Scientific Society-IMACSSS, 35-959 Rzeszow, A. Towarnickiego Str. 3/17A,

Poland

4. Faculty of Physical Education, University of Rzeszów, Rzeszow 35-959, Poland

5. Department of Physical and Sport Education, Faculty of Physical and Sport Sciences, University of León, Vegazana Campus, 24071-León, Spain

\begin{abstract}
Aging leads to a general decline in body functions that affects quality of life. Sports practice is recommended as a means to attenuate the impact of the aging process. The aim of this study was to identify and analyze the neuromuscular activity and its patterns on active karate practitioners and assess the effects of the aging process. Two groups of black belt male karate practitioners, one with nine practitioners over 50 years old and the other with 21 practitioners aged between 20-30 years old performed the frontal kick mae-geri. Kinematic and electromyographic data of five muscles were collected from the right leg. Results showed two distinct periods of muscular activation in the mae-geri performance, with similar muscles activity patterns between groups. Nevertheless, some differences were found in the muscles' activity onset and offset, in root mean square and in inter-muscle delay and co-contraction periods. Those differences indicated that aging affects the neuromuscular activity in the mae-geri performance of the oldest karate practitioners. Nevertheless, the results showed that lifelong practice of karate can preserve the quality of the neuromuscular activity in older practitioners when they perform a kick, and this could be associated with benefits to balance control.
\end{abstract}

Key words: Aging, motor control, neuromuscular activity, karate, combat sports, martial arts.

\section{Introduction}

After the third decade of life, humans start to experience an aging-related decline in biological functions that affect the locomotors' performance [1], compromising their ability to do normal activities and to maintain the practice of sport. Those declines happen at the level of muscular strength production [2, 3 ], with a decrease of maximal strength associated with loss of muscle mass, and atrophy, with a decrease in cross-sectional area of muscle fibers and a decrease in the number of muscle fibers [4-6]. Age-related

Corresponding author: António Manuel Vitória Vences de Brito, Ph.D., professor, research fields: kinesiology, neuromuscular control and coordination, physiology and injuries in sport. E-mail: abrito@esdrm.ipsantarem.pt. reduction of strength also depends on the decrease in mitochondria and mitochondrial DNA [7], with a reduction of synthesis of muscle proteins [8].

The neurological activity declines with aging, too $[9,10]$. At this level, changes occur in the detection of visual, vestibular and somatosensory stimuli [11-13], in processing the information [14] and in the response through muscle contraction [15]. These changes are related to the loss of neurons and a decrease in the functional capacity of the remaining neurons, leading to a decrease in the velocity of conduction in the nerve [16-19]. Proprioceptive receptors related with the control and determination of the limbs' position and movements continue to deteriorate with age, leading to a decrease in neuromuscular control and increasing 
the risk of falls in the elderly $[9,20]$.

In association with neuromuscular control and strength production, other factors, such as longer co-activation and co-contraction [21], or the reduction of specific tension in muscles may contribute to the age-related loss of force [22]. The co-activation and co-contraction between agonist and antagonist muscles are higher in the earlier ages, decreasing progressively with maturation, and learning and practice of skills [23]. There is a stabilization period [24], but the co-activation and co-contraction increases with aging, fundamentally after the fifth decade of life [25-27].

Neuromuscular control patterns of the movements and the changes related with aging lead to changes in the performance of both simple and complex skills. Several studies concluded that the changes in movement time increase with aging [10, 14, 28, 29], but the older people can perform motor skill activities using similar control strategies to young adults [28]. Rand and Stelmach [14] showed that several movement components were differently affected by aging, and this affected in different ways the decrease of the overall performance. Those authors suggest the existence of an age-related deficit in processing of sensory information, reducing the capability to plan and organize sequential actions.

The decline in neuromuscular function related to age $[30,31]$ can be slowed or delayed with regular physical activity [6]. Studies about training in older people confirm improvements in muscle strength [3, 32, 33], muscle power [34], explosive strength, walking speed and in balance. Improvements in the maximum oxygen consumption [1], as in the mitochondria functions [7] were found, which is important for the contractile activity of muscles.

Martial arts allow practitioners to maintain a regular and continuous practice throughout life, and some studies related these practices with benefits in the aging process. Gatts and Woollacott [35] showed that
Tai Chi practice improves neuromuscular control, which improves the balance and reduces the risk of falls. Similar conclusions about balance control were found in the Korean martial art Taekwondo [36], and also improvements in muscular strength and flexibility were noted [37].

In karate, the performance of specific skills is dependent on a high level of control and coordination of the limbs movements and balance, which is dependent on effective neuromuscular control. Consequently, aging can bring some problems to karate practitioners during the performance of their skills.

The latest research in karate has mainly focused on the kinesiological aspects of skills performance in both regular and elite practitioners aged below 50 years old. Some researchers analyzed the neuromuscular patterns, kinematic and kinetic activity of the punches [38-40], and others the kicks [41-45]. However, research about the relationship between aging and karate practice has to be done. Thus, considering the karate with a practice possible for life, we must know and understand how the aging process affects it, particularly in terms of neuromuscular activity, and the potential benefits to the quality of life of its practitioners.

The purpose of this study was to identify and compare the neuromuscular patterns of activity in veteran and younger black belt karate practitioners during the performance of the frontal kick mae-geri to verify the existence of differences related to aging. To fulfill this aim, the activity of the muscles of the hip flexion, knee extension and foot plantar flexion were compared between the younger and veteran groups during the performance of the mae-geri kick. We hypothesized that continuous and regular karate practice will reduce the effects of aging process on neuromuscular activity in older karate practitioners, preventing and attenuating the decrease in neuromuscular activity. 
Table 1 Physical characterization of VetK and YgK practitioners.

\begin{tabular}{lllllllll}
\hline & $\begin{array}{l}\text { Age } \\
(\text { years })\end{array}$ & $\begin{array}{l}\text { Height } \\
(\mathrm{cm})\end{array}$ & $\begin{array}{l}\text { Weight } \\
(\mathrm{kg})\end{array}$ & $\begin{array}{l}\text { Leg length } \\
(\mathrm{cm})\end{array}$ & $\begin{array}{l}\text { Body fat } \\
(\%)\end{array}$ & $\begin{array}{l}\text { Weekly training hours } \\
(\mathrm{h})\end{array}$ & $\begin{array}{l}\text { Black belt level } \\
(\text { Dan })\end{array}$ & $\begin{array}{l}\text { Years of } \\
\text { practice }\end{array}$ \\
\hline VetK & $54 \pm 4^{\mathbf{a}}$ & $176 \pm 5$ & $76 \pm 9$ & $91 \pm 3$ & $19 \pm 2^{\mathbf{a}}$ & $5 \pm 1$ & $5 \pm 1^{\mathbf{a}}$ & $35 \pm 10^{\mathbf{a}}$ \\
YgK & $23 \pm 5$ & $172 \pm 7$ & $72 \pm 14$ & $89 \pm 5$ & $13 \pm 7$ & $7 \pm 4$ & $1 \pm 1$ & $13 \pm 4$ \\
\hline
\end{tabular}

VetK = Veteran karate practitioners. YgK = Young karate practitioners. Values are means \pm SD.

${ }^{\text {a }}$ Specifies statistically significant differences between groups $(\alpha \leq 0.05)$.

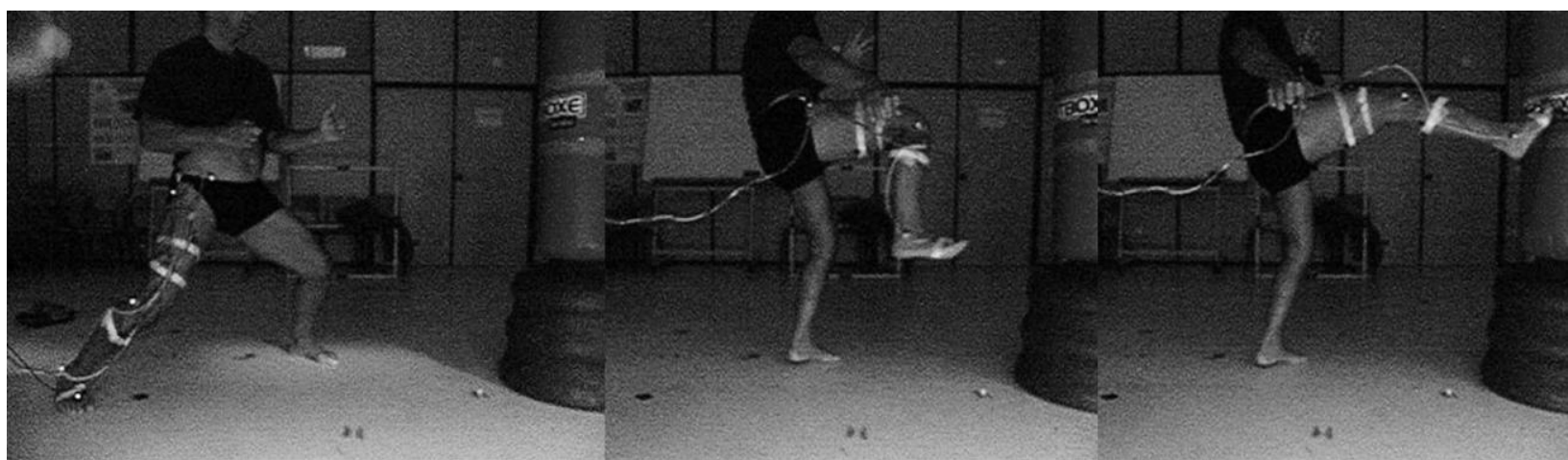

Fig. 1 Illustration of the experimental setup showing a veteran karateka facing the bag at the starting position of zenkutsu-dachi, with the right leg in backward extension, and the left leg flexed on the thigh and bent at the knee, with both feet on the floor, and the mae-geri kick movement until the final position of the foot in contact with the target.

\section{Methods}

\subsection{Participants}

Thirty male black belt karate practitioners with more than 10 years of continuous practice volunteered to participate in the study. Two groups were formed; one with nine veteran karate practitioners aged more than 50 years old (VetK), and the other with 21 karate practitioners aged between 20 and 30 years old (YgK).

All participants were in perfect health and without history of neuromuscular or cardiovascular disease. All participants signed an informed consent document.

The demographics of the two groups are shown in Table 1.

This study was conducted according to the Helsinki Declaration and was approved by the scientific committee of the Sport Sciences School of Rio Maior, Polytechnic Institute of Santarém, Portugal.

\subsection{Experimental Design}

Each karate practitioner (karateka) participated in a single measurement session where the surface electromyographic (SEMG) activity of five muscles and the kinematic of the right leg (dominant) during the performance of the mae-geri kick were recorded simultaneously. The kick was performed into a vertical floor training bag. The target area was at a height of $90 \mathrm{~cm}$ from the ground and at a distance of each participant's lower limb length. Participants started from the typical karate static stance of zenkutsu-dachi (Fig. 1). They kicked in response to a sound stimulus activated by a trigger linked and synchronized between the SEMG and the video recording device. Each participant performed three fast and strong repetitions of the mae-geri kick with impact of the metatarsals, koshi, on the bag. There was a rest period of 30 seconds between repetitions. The median value of the three trials was used for analysis.

\subsection{Apparatus}

After skin preparation, electrodes were placed on the muscles according to the recommendation by SENIAM [46], with the ground electrode placed on the fifth lumbar vertebra. The SEMG was recorded through the MP100 Data Acquisition System with a sampling rate of $1050 \mathrm{~Hz}$, and active bipolar surface electrodes (centres separated by $20 \mathrm{~mm}$ ), TSD Model 150TM (BIOPAC Systems, Santa Barbara, CA, USA), 
with an input impedance of $10 \mathrm{G} \Omega$, noise of $1 \mu \mathrm{V}$, common mode rejection ratio (CMRR) of $95 \mathrm{db}$, and gain of 1000 .

The kick movement was filmed with a high-speed camera (Casio EX-FH20), with a sampling frequency of $210 \mathrm{~Hz}$, and positioned perpendicular to the plane of movement at a distance of two meters. Five reflective markers were placed along the lower extremity, specifically on the right anterior superior iliac spine, the prominence of the greater trochanter, the lateral condyle of the femur, the fibular malleolus and the dorsal facet of the second metatarsal head.

The records were cut and analyzed with the software Ariel Performance Analysis System (APAS, Ariel Dynamics-2003). The criterion for cutting the raw data of SEMG and video records for analysis was to carry out the initial cut $200 \mathrm{~ms}$ before the trigger input signal and the final cut $200 \mathrm{~ms}$ after the moment the koshi made contact with the bag. The first frame where koshi contacts in the bag was considered as the instant time 0 for all measurements.

On the final files the determination of the onset/offset on muscle activation and the onset of segmental movements were done visually by a single expert researcher on the Matlab outputs. The remaining data were automatically processed through the Matlab software.

\subsection{Data Reduction}

\subsubsection{SEMG}

The SEMG was recorded from Quadriceps Femoris in the portions of the Rectus Femoris (RF) and Vastus Lateralis (VL), the long head of the Biceps Femoris (BF) muscle, the Tibialis Anterior (TA) muscle and the lateral portion of the Gastrocnemius (GA) muscle.

The SEMG signals were digitally filtered (10 to 400 $\mathrm{Hz}$ ), full wave rectified, smoothed with a low pass filter of $12 \mathrm{~Hz}$ (Butterworth, 4th order), and normalized using the average value of the maximum peak of electromyographic activity of the three runs [47, 48], in each studied muscle (Fig. 2).

In the isolated SEMG bursts of each trial, the dependent variables of onset and offset of muscle activation, muscle activity duration, root mean square (RMS), the delay time between the beginning of inter-muscles activity and the co-contraction time between muscles were processed and studied.

To process the inter-muscles delay (IMD) and the co-contraction time, the muscles were organized in pairs with respect to their action as agonists (RF, VL, GA) and antagonists (BF, TA) on the joint movements (hip, knee and ankle).

\subsubsection{Kinematic}

The beginning of the forward movement (onset) in hip, knee, ankle and foot were the variables considered

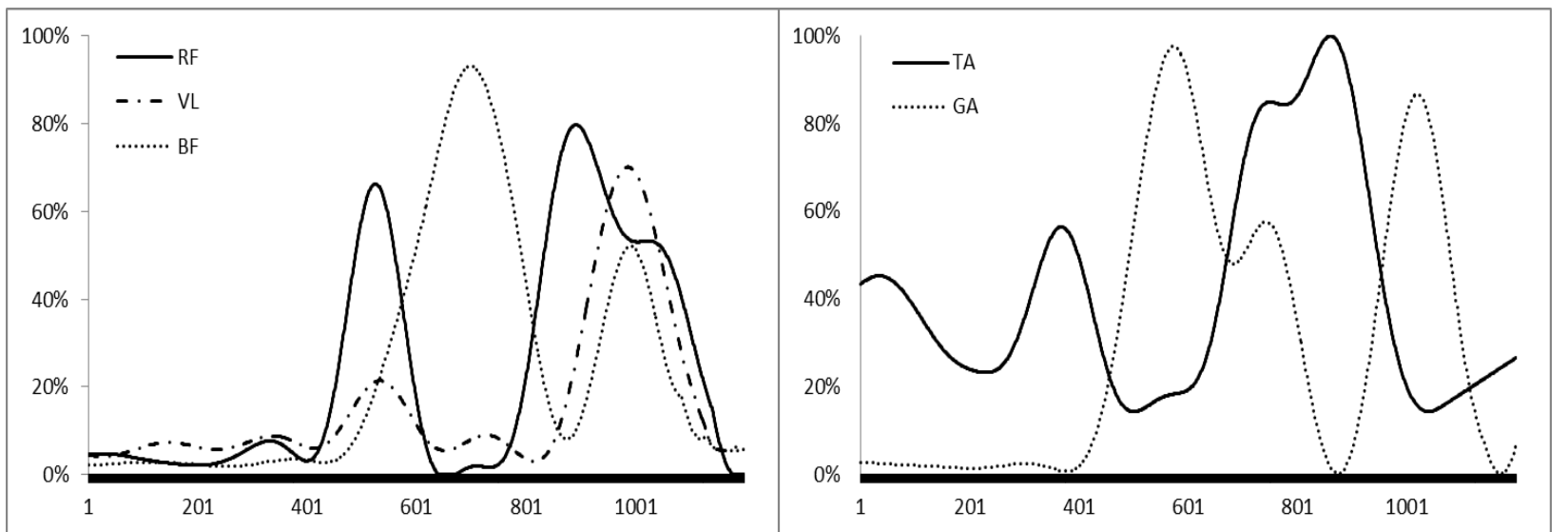

Fig. 2 Illustration of the SEMG of the Rectus Femoris (RF) and Vastus Lateralis (VL), the long head of the Biceps Femoris (BF), Tibialis Anterior (TA), and the lateral portion of the Gastrocnemius (GA) muscles activity during the mae-geri performance. 
in this study. They were computed through the APAS system and the Matlab.

The virtual lab space was calibrated using eight control points, identified by a number and respective coordinates $(x, y)$, in order to identify in all frames the coordinates of the reflective markers fixed on the participants. Then the reconstruction of the movement trajectory was made. The initial data were smoothed by the APAS software with a low pass digital filter of $5 \mathrm{~Hz}$ cut off [49].

\subsection{Statistics}

Descriptive data are presented as means and standard deviations. Normality of the data distribution (Kolmogorov-Smirnov) and homogeneity of variances (Levene's test) was evaluated for all variables. One Way ANOVA was performed to examine the differences between the two groups on the SEMG dependent variables and kinematic. Level of statistical significance was set at $\alpha \leq 0.05$.

Statistical analysis was performed with Statistical Package for the Social Sciences (SPSS 20.0 for Windows ${ }^{\circledR}$, SPSS Inc, Chicago, USA).

\section{Results}

\subsection{Demographic Parameters}

There were significant differences between VetK and $\mathrm{YgK}$ in age, body fat, black belt level and years of practice, achieving VetK higher values (Table 1).

\subsection{Segmental Movement Time}

Table 2 shows the onset time of the segmental movements as well as the significant differences between the VetK and the YgK.
The onset time of the segmental movements showed a kinematic sequence similar in both groups during the performance of the mae-geri. As is shown in the Fig 1, the kick began with a hip flexion movement followed by a proximal to distal and back forward direction until the koshi (metatarsals) contacted the training bag.

In VetK, all the mechanic segmental movement of mae-geri kicking began about $432 \mathrm{~ms}$ before the contact of the koshi with the bag. The last segment to initiate the movement was the metatarsal, $259 \mathrm{~ms}$ before the contact. All the segmental movements began significantly earlier in $\mathrm{YgK}(\alpha<0.05)$.

\subsection{Muscle Activity}

As is shown in the Fig 2, two different activations occurred after the trigger signal, separated by an electrical silence were identified in the SEMG files, in all studied muscles and in both groups. For each muscle these activations were referenced as first (e.g. RF1 or (1)) and second (e.g. RF2 or (2)) activation period. Other studies have also showed a double muscular activation in karate $[39,50]$ and elite mixed martial arts fighters [51].

Table 3 shows the SEMG dependent variables to the muscle activity in the VetK and Ygk and the significant differences between groups.

The onset of muscle activation defines a sequential time pattern of muscle recruitment. In VetK, the TA and the RF were the earliest muscles to be activated during the first period of activity (TA1 and RF1 at 616 $\mathrm{ms}$ before contact in the bag). Next, the VL and BF activated, and finally the GA activated. The second period of activation had a similar sequence, except that the GA was activated before the BF.

Table 2 Kinematic time variables between VetK and YgK practitioners.

\begin{tabular}{|c|c|c|c|c|c|}
\hline & & Hip & Knee & Ankle & Foot \\
\hline \multirow{2}{*}{ Onset movement time (ms) } & VetK & $432 \pm 43^{a}$ & $334 \pm 46^{a}$ & $296 \pm 18^{a}$ & $259 \pm 11^{a}$ \\
\hline & YgK & $495 \pm 71$ & $406 \pm 48$ & $353 \pm 38$ & $309 \pm 31$ \\
\hline
\end{tabular}

VetK = Veteran karate practitioners. YgK = Young karate practitioners. Values are means \pm SD. Time-millisecond.

${ }^{a}$ Specifies statistically significant differences between groups $(\alpha \leq 0.05)$. 
Table 3 The variables of muscle SEMG in the mae-geri performance by VetK and YgK practitioners.

\begin{tabular}{|c|c|c|c|c|c|c|c|c|c|c|c|}
\hline & & RF1 & VL1 & BF1 & TA1 & GA1 & RF2 & VL2 & BF2 & TA2 & GA2 \\
\hline \multirow{2}{*}{$\begin{array}{l}\text { Onset muscles } \\
\text { activation time } \\
\text { (ms) }\end{array}$} & VetK & $616 \pm 62^{a}$ & $558 \pm 56^{a}$ & $499 \pm 61^{a}$ & $616 \pm 61$ & $479 \pm 43$ & $255 \pm 53$ & $135 \pm 26^{a}$ & $93 \pm 35$ & $325 \pm 44$ & $114 \pm 38$ \\
\hline & YgK & $513 \pm 69$ & $500 \pm 70$ & $411 \pm 61$ & $573 \pm 101$ & $465 \pm 42$ & $241 \pm 59$ & $155 \pm 24$ & $87 \pm 46$ & $293 \pm 70$ & $110 \pm 32$ \\
\hline \multirow{2}{*}{$\begin{array}{l}\text { Offset muscles } \\
\text { activation time } \\
\text { (ms) }\end{array}$} & VetK & $395 \pm 65^{a}$ & $367 \pm 55^{a}$ & $219 \pm 69^{a}$ & $414 \pm 70^{a}$ & $187 \pm 48$ & $-22 \pm 28$ & $-45 \pm 18$ & $-44 \pm 15$ & $62 \pm 18^{a}$ & $-56 \pm 12$ \\
\hline & YgK & $322 \pm 64$ & $318 \pm 60$ & $110 \pm 64$ & $302 \pm 85$ & $167 \pm 42$ & $-31 \pm 28$ & $-47 \pm 18$ & $-36 \pm 23$ & & \\
\hline \multirow{2}{*}{$\begin{array}{l}\text { Muscle } \\
\text { activity } \\
\text { duration (ms) }\end{array}$} & VetK & $217 \pm 57$ & $196 \pm 48$ & $273 \pm 98$ & $193 \pm 43$ & $304 \pm 72$ & $278 \pm 59$ & $178 \pm 23^{a}$ & $127 \pm 44$ & $265 \pm 39$ & $154 \pm 21$ \\
\hline & $\mathrm{YgK}$ & $183 \pm 43$ & $179 \pm 34$ & $292 \pm 79$ & $256 \pm 95$ & $299 \pm 54$ & $271 \pm 47$ & $201 \pm 22$ & $134 \pm 51$ & $262 \pm 53$ & $167 \pm 23$ \\
\hline \multirow{2}{*}{$\begin{array}{l}\text { SEMG root } \\
\text { mean square } \\
\text { (\% RMS) }\end{array}$} & VetK & $15 \pm 2^{a}$ & $24 \pm 8$ & $51 \pm 5^{a}$ & $28 \pm 13$ & $43 \pm 7$ & $57 \pm 7^{a}$ & $59 \pm 5$ & $29 \pm 9$ & $59 \pm 7$ & $47 \pm 9$ \\
\hline & YgK & $28 \pm 13$ & $32 \pm 14$ & $41 \pm 10$ & $29 \pm 12$ & $46 \pm 9$ & $49 \pm 9$ & $56 \pm 7$ & $35 \pm 13$ & $54 \pm 11$ & $47 \pm 10$ \\
\hline
\end{tabular}

VetK = Veteran karate practitioners. YgK = Young karate practitioners. Values are means \pm SD. Time-millisecond; RMS—\% of the normalized SEMG; RF-Rectus Femoris; VL-Vastus Lateralis; BF-Biceps Femoris; TA-Tibialis Anterior; GE-Gastrocnemius. First (1) and second (2) activation periods in the studied muscles.

${ }^{a}$ Specifies statistically significant differences between groups $(\alpha \leq 0.05)$.

Table 4 Inter-muscles delay and co-contraction in the execution of mae-geri by VetK and YgK practitioners.

\begin{tabular}{llllllllll}
\hline & & RF1-VL1 & RF1-BF1 & VL1-BF1 & TA1-GA1 & RF2-VL2 & RF2-BF2 & VL2-BF2 & TA2-GA2 \\
\hline IMD & VetK & $64 \pm 58^{\text {a }}$ & $123 \pm 108$ & $89 \pm 64$ & $146 \pm 53$ & $113 \pm 41$ & $152 \pm 45$ & $33 \pm 50^{\mathbf{a}}$ & $218 \pm 59$ \\
$(\mathrm{~ms})$ & YgK & $29 \pm 27$ & $123 \pm 74$ & $96 \pm 76$ & $121 \pm 71$ & $84 \pm 54$ & $146 \pm 67$ & $75 \pm 44$ & $183 \pm 69$ \\
Co-contraction & VetK & $153 \pm 63$ & $89 \pm 115$ & $111 \pm 81$ & $40 \pm 44^{\text {a }}$ & $157 \pm 27^{\text {a }}$ & $118 \pm 56$ & $140 \pm 46$ & $45 \pm 23$ \\
$(\mathrm{~ms})$ & YgK & $179 \pm 49$ & $88 \pm 116$ & $99 \pm 100$ & $153 \pm 122$ & $188 \pm 37$ & $105 \pm 51$ & $128 \pm 44$ & $76 \pm 50$ \\
\hline
\end{tabular}

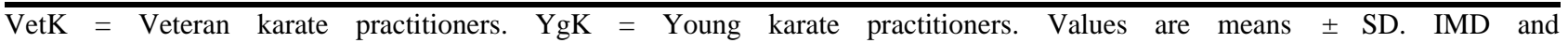
co-contraction-millisecond; RF-Rectus Femoris; VL—Vastus Lateralis; BF-Biceps Femoris; TA-Tibialis Anterior; GA-Gastrocnemius. First (1) and second (2) activation periods in the studied muscles.

${ }^{a}$ Specifies statistically significant differences between groups $(\alpha \leq 0.05)$.

The YgK presented a similar sequence time of muscles activation, however, during the first period the $\mathrm{VL}$ and $\mathrm{BF}$ muscles were activated significantly later than in the VetK and in the second period the VL was activated significantly before than the VetK (Table 3).

The offset time in the VetK presented a similar sequence to the onset but in the second period the muscles activity finished after the contact of the koshi with the bag, with exception of the TA (Table 3). In the VetK, the BF was deactivated before the VL. The YgK differed significantly because their muscles deactivate closer to contact of the koshi with the bag, specifically the RF, VL, BF and TA in the first activity and the TA in the second activity.

The duration time of musculars activity in the VetK varies between $304 \mathrm{~ms}$ (GA1) and $193 \mathrm{~ms}$ (TA1) in the first activation period and between $278 \mathrm{~ms}$ (RF2) and $127 \mathrm{~ms}$ (BF2) in the second activation period.
Nevertheless, the veterans' GA, BF and VL muscles had a larger time of activity in the first period than in the second (between 304 and $196 \mathrm{~ms}$ ), unlike the RF and TA which presented a larger time of activity in the second period (278 and $265 \mathrm{~ms}$, respectively). YgK showed a significantly larger duration time of activation in VL muscle during the second period.

The muscle activation intensity in the VetK had a minimum value of RMS of $15 \%$ on the RF and a maximum value of $51 \%$ on the $\mathrm{BF}$ in the first period of activity. In the second period of activity, the muscles increased the RMS about 30\%, except for the $\mathrm{BF}$ where the intensity was reduced about $20 \%$ (Table 3). YgK had significantly higher RMS in RF muscle and lower in BF muscle during the first activity. In the second activity, the RMS of the RF muscle was lower in the $\mathrm{YgK}$.

Table 4 shows the IMD and co-contraction time between muscles in the VetK and $\mathrm{YgK}$ as well as the 
significant differences between groups.

The VetK presented higher IMD period in VL-BF muscles, respectively agonist and antagonist of the knee extension, during the first activity than in the second. The RF-VL, agonists of hip flexion and knee extension, the RF-BF, respectively agonist and antagonist of knee extension, and the TA-GA, respectively antagonist and agonist of foot plantar flexion, had a lower IMD period during the first activity, but in the TA-GA the IMD was higher than in the RF-VL and RF-BF in the two activation periods. YgK showed significantly smaller IMD in RF-VL and higher in VL2-BF2 than did the VetK (Table 4).

The co-contraction time in the VetK was higher for all the muscle pairs in the second period of activation, particularly on RF-BF $((1)=89 \mathrm{~ms} ;(2)=118 \mathrm{~ms})$ and $\operatorname{VL}-B F((1)=111 \mathrm{~ms} ;(2)=140 \mathrm{~ms})$, with an increase about $30 \mathrm{~ms}$ on this period. TA-GA first period of co-contraction and RF-BF second period of co-contraction were significantly higher in $\mathrm{YgK}$ than in VetK (Table 4).

\section{Discussion}

In the present study, the results showed the existence of a specific neuromuscular phasic pattern of control and coordination of the mae-geri kick performance, on both groups, which seems to be adapted to the age of the karate practitioners.

The complexity of the kick performance generated two distinct contractile activities on the muscles in all karate practitioners, much as was found in other studies [45, 51]. The first activation was related with the initial hip flexion movement and the stabilization of the knee and ankle, while the second activation was related with the knee extension and foot plantar flexion at the ankle level to finalize the kick. In these muscles, there was found to be a temporal and reciprocal sequential order of agonists and antagonists recruitment. This order was similar between the two groups, and is typical of ballistic movements, corresponding to a proximal-to-distal mechanic segmental sequence of movements $[39,45,50]$. The physical action was similar between groups, but the movements began earlier in the YgK. Nevertheless, the muscle activation on $\mathrm{YgK}$, especially in the RF1, VL1, BF1 and VL2, began closer to the moment of contact of the koshi with the bag, reflective of less time being required to transfer the contractile muscle energy to the segments, to move and control them during the kick. This shows that in VetK, there could exist a loss of conduction velocity by the nervous system, as evidenced by some authors [16-19], and a loss of motoneurons and muscle fibers as a consequence of the natural aging process $[19,30]$. However, these results could as well be related to the veterans' significantly higher body fat and less muscular mass, resulting in a smaller number of fibers being available [2-6] in the VetK, probably with a reduction in the synthesis rates of muscle proteins and mitochondrial functions [8].

The neuromuscular conduction velocity and the characteristics of muscles seems to be two primary factors involved in the differences between VetK and YgK. The study shows that during the muscles' activity duration, the agonist muscles VL and GA, and the antagonist muscle $\mathrm{BF}$, tend to be activated for more time in the first period than in second period, in the VetK. This shows the importance of the initial control of the acceleration and balance when the karateka begins to perform the kick from a static stance [45]. In the second period, there is an increase in the duration of the RF activation. This is associated with the continuity of hip flexion and the beginning of knee extension, and with the TA activation, to stabilize the foot position. YgK tend to have a higher second period of activity in the agonist (RF and VL) and antagonist (TA) muscles, which might reflect that the second activation increases the acceleration and velocity of knee flexion and the foot stabilization, in anticipation of contact with the bag. In any event, the activity duration of the VL muscle during the second period was significantly higher in the $\mathrm{YgK}$. 
The RMS shows that the muscle activation intensity was, in general, lower than $60 \%$. Consequently, there was not necessarily a great intensity of muscular activity needed to perform the mae-geri kick. In this context, the activation intensity of the agonist (RF, VL and GA) and antagonist (TA) muscles was lower in the first period than in the second period, in both groups, except in the BF muscle. This reflects that the main purpose of the first muscles' activity was to begin the segmental movements, with the hip and knee well controlled by the action of the BF muscle, without great levels of strength to accelerate the segments, as was referred by other study [45]. Despite this common behavior in both groups, significant difference was found between VetK and $\mathrm{YgK}$ in the activation intensity of both $\mathrm{RF}$ and $\mathrm{BF}$ muscles, during both periods of activity. This could reflect a greater intervention of the antagonist action (BF) in the hip and knee during the initial movement of the kick to increase the control, stability and body balance in the VetK.

The IMD shows if the muscles were simultaneously activated. The IMD in the mae-geri performance tends to be lower in the first period of muscles activity than in the second period, in both groups, except in VL2-BF2. This lower IMD shows that the antagonist activity in the first period is needed to control the initial segmental movements and transfer the body weight from a bipedal stance into a unipedal stance. This action needs joints to stabilize and requires balance control. In the second period, the antagonists were activated later, facilitating the initial acceleration and velocity in the joint movements, and preparing the segments for the impact of the koshi on the bag.

VetK tended to have a higher IMD during both periods of muscles activity than $\mathrm{YgK}$, especially in RF1-VL1. The RF1-VL1 differences in the IMD between the groups could be related with the anticipation in the transfer of contractile energy to the knee extension in the YgK. In the VetK it must be initially more centered in the joint stabilizations and balance control, and this is something associated with the aging effects [21, 25-27, 35, 36]. This requirement was reflected in a lower IMD of the RF2-BF2 in the VetK, who, because they needed the antagonist muscle BF2 activity to increase the knee extension control, slowed down the movement but increased the control of the impact and body balance.

The typical phasic pattern of reciprocal enervation in the agonist and antagonist muscles recruitment was associated with well-trained and learned ballistic skills. In the performance of those skills, the co-contraction periods tended to be shorter. Nevertheless, the aging process affected the neuromuscular control patterns, namely, increasing the agonist and antagonist muscles co-contraction in older people [21, 25-27]. In the present study, the times of muscular co-contraction was similar between the two groups, and tended to be greater during the second period of activity. This reflected an increase of control over the joint movements to prepare the contact of the koshi with the bag. However, the VetK presented significantly smaller co-contraction times in the TA1-GA1 and RF2-VL2. Thus, the co-contraction and IMD data suggest that the effects of the aging process could be seen in the middle-aged VetK, in patterns typical to co-contraction found in elderly people. The longevity of the karate practice could be the reason to the maintenance of this similar pattern between VetK and YgK.

In general, it is assumed that aging alters the neuromuscular activity, and possibly, the motor control strategies, leading to performance deficits, and it was our expectation to find significantly different SEMG parameters, allowing the identification of the aging process in the neuromuscular patterns on VetK. Nonetheless, we found a specific neuromuscular pattern to control and coordinate the mae-geri kick performance that was similar between VetK and $\mathrm{YgK}$. The few differences found in temporal muscular activity of the VetK showed the effects of aging, but the continued practice over the years, presumably 
increased efficiency for this group, and that could explain the similar neuromuscular control activity with the $\mathrm{YgK}$.

This study was limited to a sample of high rank karate practitioners, all male, all black belts, and all with many years of practice. This evidence leads to the question whether the middle aged practitioner and youths, women, or lower level practitioners would show the same characteristics of neuromuscular pattern in the kicking control. Nonetheless, the results of this study provide useful information to karate practitioners and coaches, and also to researchers who study the neuromuscular control in sportive skills performance related with aging.

\section{Conclusions}

In conclusion, the VetK showed that continuous practice throughout life can preserve the quality of the neuromuscular activity in the control of kicking performance. In fact, the Vetk and YgK show a similar pattern for controlling and coordinating their muscles when they perform the mae-geri kick. However, the older practitioners show a few adaptations in the onset time to the recruitment of hip muscles. This could be related to a loss of transmission velocity in the nervous system and a loss in the excitation capacity and contractile capacity of the muscles due to aging.

The similar intensity of muscular activity, with the exception of the Rectus Femoris and Biceps Femoris, and the similar times of intermuscular delay and co-contraction periods in almost all of the pairs of analyzed muscles in the two groups, can also confirm the conclusion that Karate practice by elderly people mitigates the aging effects and can contribute to a better quality of life.

The presented and discussed findings confirm the formulated hypothesis that continuous and regular karate practice will reduce the effects of aging process on neuromuscular activity in older karate practitioners, preventing and attenuating the decrease in neuromuscular activity. So it is reasonable to assume that the improvement of body balance and the prevention of falls are important benefits of karate practice by middle-aged and elderly people, and that continuous Karate practice might be beneficial for the functionality of neuromuscular activity.

\section{Acknowledgments}

The authors want to thank all the karate practitioners who voluntarily participated. The authors want to thank to the martial arts researcher Joseph $\mathrm{R}$. Svinth for reviewing the written English, and also the Karate Association of Setubal District (AKDS) for its support to this research.

\section{References}

[1] Holviala, J., Kraemer, W. J., Sillanpää, E., Karppinen, H., Avela, J., Kauhanen, A., Häkkinen, A., and Häkkinen, K. 2012. "Effects of Strength, Endurance and Combined Training on Muscle Strength, Walking Speed and Dynamic Balance in Aging Men.” European Journal of Applied Physiology 112 (4): 1335-47.

[2] Häkkinen, K. 1994. "Neuromuscular Adaptation during Strength Training, Aging, Detraining and Immobilization: A Review." Critical Reviews in Physical and Rehabilitation Medicine 6: 161-98.

[3] Jozsi, A. C., Campbell, W. W., Joseph, L., Davey, S. L., and Evans, W. J. 1999. "Changes in Power with Resistance Training in Older and Younger Men and Women." The Journals of Gerontology: Series A, Biological Sciences and Medical Sciences 54 (11): M591-6.

[4] Brooks, S. V., and Faulkner, J. A. 1994. "Skeletal Muscle Weakness in Old Age: Underlying Mechanisms." Medicine and Science in Sports and Exercise 26 (4): 432-9.

[5] Doherty, T. J., Vandervoort, A. A., Taylor, A. W., and Brown, W. F. 1993. "Effects of Motor Unit Losses on Strength in Older Men and Women." Journal of Applied Physiology 74 (2): 868-74.

[6] Faulkner, J. A., Larkin, L. M., Claflin, D. R., and Brooks, S. V. 2007. "Age-Related Changes in the Structure and Function of Skeletal Muscles." Clinical and Experimental Pharmacology and Physiology 34 (11): 1091-6.

[7] Eluamai, A., and Brooks, K. 2013. "Effect of Aerobic Exercise on Mitochondrial DNA and Aging." Journal of Exercise Science and Fitness 11 (1): 1-5. 
[8] Barazzoni, R, Short, K. R., and Nair, K. S. 2000. "Effects of Aging on Mitochondrial DNA Copy Number and Cytochrome C Oxidase Gene Expression in Rat Skeletal Muscle, Liver, and Heart." The Journal of Biological Chemistry 275 (5): 3343-7.

[9] Horak, F. B. 2006. "Postural Orientation and Equilibrium: What Do We Need to Know about Neural Control of Balance to Prevent Falls?" Age and Ageing 35 (S2): ii7-11.

[10] Jiménez-Jiménez, F. J., Calleja, M., Alonso-Navarro, H., Rubio, L., Navacerrada, F., Pilo-de-la-Fuente, B., Plaza-Nieto, J. F., Arroyo-Solera, M., García-Ruiz, P. J., García-Martín, E., and Agúndez, J. A. 2011. "Influence of Age and Gender in Motor Performance in Healthy Subjects." Journal of the Neurological Sciences 302 (1-2): 72-80.

[11] Lord, S. R. 2006. "Visual Risk Factors for Falls in Older People." Age and Ageing 35 (S2): ii42-5.

[12] Matheson, A. J., Darlington, C. L., and Smith, P. F. 1999. "Dizziness in the Elderly and Age-Related Degeneration of the Vestibular System." New Zealand Journal of Psychology 28 (1): 10-6.

[13] Whipple, R., Wolfson, L., Derby, C., Singh, D., and Tobin, J. 1993. "Altered Sensory Function and Balance in Older Persons." Journal of Gerontology 48 (Sep): 71-6.

[14] Rand, M. K., and Stelmach, G. E. 2012. "Effect of Aging on Coordinated Eye and Hand Movements with Two-Segment Sequence." Motor Control 16 (4): 447-65.

[15] Kenney, W. L., Wilmore, J., and Costill, D. 2012. Physiology of Sport and Exercise (5th ed.). Champaign: Human Kinetics.

[16] Jagga, M., Lehri, A., and Verma, S. K. 2011. "Effect of Aging and Anthropometric Measurements on Nerve Conduction Properties-A Review." Journal of Exercise Science and Physiotherapy 7 (1): 1-10.

[17] Sato, A., Sato, Y., and Suzuki, H. 1985. "Aging Effects on Conduction Velocities of Myelinated and Unmyelinated Fibers of Peripheral Nerves." Neuroscience Letters 53 (1): 15-20.

[18] Stetson, D. S., Albers, J. W., Silverstein, B. A., and Wolfe, R. A. 1992. "Effects of Age, Sex, and Anthropometric Factors on Nerve Conduction Measures." Muscle and Nerve 15 (10): 1095-104.

[19] VanPutte, C. L., Regan, J. L., Russo, A. F., Seeley, R. R., Stephens, T. D., and Tate, P. 2014. Seeley's Anatomy and Physiology (10th ed.). London: McGraw-Hill.

[20] Proske, U., and Gandevia, S. C. 2012. "The Proprioceptive Senses: Their Roles in Signaling Body Shape, Body Position and Movement, and Muscle Force." Physiological Reviews 92 (4): 1651-97.

[21] Kellis, E., Arabatzi, F., and Papadopoulos, C. 2003. "Muscle Co-activation around the Knee in Drop Jumping
Using the Co-contraction Index." Journal of Electromyography and Kinesiology 13 (3): 229-38.

[22] Klein, C. S., Rice, C. L., and Marsh, G. D. 2001. "Normalized Force, Activation, and Coactivation in the Arm Muscles of Young and Old Men." Journal of Applied Physiology 91 (3): 1341-9.

[23] Basmajian, J. V. 1997. "Motor Learning and Control: A Working Hypothesis." Archives of Physical Medicine and Rehabilitation 58 (1): 38-41.

[24] Vandervoort, A. A. 2002. "Aging of the Human Neuromuscular System." Muscle and Nerve 25 (1): 17-25.

[25] Bautmans, I., Vantieghem, S., Gorus, E., Grazzini, Y. R., Fierens, Y., Pool- Goudzwaard, A., and Mets, T. 2011. "Age-related Differences in Premovement Antagonist Muscle Co-activation and Reaction-time Performance." Experimental Gerontology 46 (8): 637-42.

[26] Franz, J. R., and Kram, R. 2012. "How Does Age Affect Leg Muscle Activity/Coactivity during Uphill and Downhill Walking?" Gait and Posture, 37, 378-384. doi: 10.1016/j.gaitpost.2012.08.004.

[27] Peterson, D. S., and Martin, P. E. 2010. "Effects of Age and Walking Speed on Coactivation and Cost of Walking in Healthy Adults." Gait and Posture 31 (3): 355-9.

[28] Buchman, A. S., Leurgans, S., Gottlieb, G. L., Chen, C. H., Almeida, G. L., and Corcos, D. M. 2000. "Effect of Age and Gender in the Control of Elbow Flexion Movements." Journal of Motor Behavior 32 (4): 391-9.

[29] Seidler, R. D., Alberts, J. L., and Stelmach, G. E. 2002. "Changes in Multi-joint Performance with Age." Motor Control 6 (1): 19-31.

[30] Kallio, J., Søgaard, K., Avela, J., Komi, P., Selänne, H., and Linnamo, V. 2012. "Age-related Decreases in Motor Unit Discharge Rate and Force Control during Isometric Plantar Flexion." Journal of Electromyography and Kinesiology 22 (6): 983-9.

[31] Mau-Moeller, A., Behrens, M., Lindner, T., Bader, B., and Bruhn, S. 2013. "Age-related Changes in Neuromuscular Function of the Quadriceps Muscle in Physically Active Adults." Journal of Electromyography and Kinesiology 23 (3): 640-8.

[32] Baker, M. K., Atlantis, E., and Fiatarone Singh, M. A. 2007. "Multi-modal Exercise Programs for Older Adults." Age and Ageing 36 (4): 375-81.

[33] Barry, B. K., and Carson, R. G. 2004. "Transfer of Resistance Training to Enhance Rapid Coordinated Force Production by Older Adults." Experimental Brain Research 159 (2): 225-38.

[34] Caserotti, P. 2010. "Strength Training in Older Adults: Changes in Mechanical Musclefunction and Functional Performance." The Open Sports Sciences Journal 3: 62-6.

[35] Gatts, S. K., and Woollacott, M. H. 2006. "Neural 
Mechanisms Underlying Balance Improvement with Short Term Tai Chi Training." Aging Clinical and Experimental Research, 18 (1): 7-19.

[36] Pons van Dijk, G., Lenssen, A. F., Leffers, P., Kingma, H., and Lodder, J. 2013. "Taekwondo Training Improves Balance in Volunteers over 40." Frontiers in Aging Neuroscience 5: 1-6.

[37] Brudnak, M. A., Dundero, D., and Van Hecke, F. M. 2002. "Are the "Hard" Martial Arts, such as the Korean Martial Art, Taekwon-do, of Benefit to Senior Citizens?" Medical Hypotheses 59 (4): 485-91.

[38] Gulledge, J. K., and Dapena, J. 2008. "A Comparison of the Reverse and Power Punches in Oriental Martial Arts." Journal of Sports Sciences 26 (2): 189-96.

[39] VencesBrito, A. M., Rodrigues Ferreira, M. A., Cortes, N., Fernandes, O., and Pezarat-Correia, P. 2011. "Kinematic and Electromyographic Analyses of a Karate Punch." Journal of Electromyography and Kinesiology 21 (6): 1023-9.

[40] Witte, K., Emmermacher, P., Hofmann, M., Schwab, K., and Witte, H. 2005. "Electromyographic Researches of Gyaku-zuki in Karate Kumite." In Proceedings of XXIII International Symposium on Biomechanics in Sports, edited by Wang, Q. Beijing, China: The People Sport Press, 861-5.

[41] Balsalobre-Fernández, C., Martínez-Majolero, V., Villacieros-Rodríguez, J., and Tejero-González, C. M. 2013. "Differences in the Vertical Jump and the Speed of Kick maegeri between International and National Karatekas." Revista de Artes Marciales Asiáticas, 8:13-20. doi: 10.18002/rama.v8i1.835. (in Spanish)

[42] Quinzi, F., Camomilla, V., Felici, F., Di Mario, A., and Sbriccoli, P. 2013. "Differences in Neuromuscular Control between Impact and no Impact Roundhouse Kick in Athletes of Different Skill Levels." Journal of Electromyography and Kinesiology 23 (1): 140-50.

[43] Quinzi, F., Sbriccoli, P., Alderson, J., Di Mario, A., and Camomilla, V. 2014. "Intra-limb Coordination in Karate
Kicking: Effect of Impacting or not Impacting a Target." Human Movement Science 33: 108-19.

[44] Quinzi, F., Camomilla, V., Felici, F., Di Mario, A., and Sbriccoli, P. 2015. "Agonist and Antagonist Muscle Activation in Elite Athletes: Influence of Age." European Journal of Applied Physiology 115 (1): 47-56.

[45] VencesBrito, A. M., Branco, M. A. C., Fernandes, R. M. C., Rodrigues Ferreira, M. A., Fernandes, O. J. S. M., Figueiredo, A. A. A., and Cynarski, W. J. 2014. "Electromyography and Cinematic Evaluation of the Kick Mae-geri in Competitive Elite Karate and Karate not Competitors Black belt." Revista UIIPS 2: 84-103. (in Portuguese)

[46] Hermens, H. J., Freriks, B., Merletti, R., Stegeman, D., Blok, J., Rau, G., Disselhorst-Klug, C., and Hagg, G. 1999. European Recommendations for Surface Electromyography: Results of the SENIAM Project. Enschede, Netherlands: Roessingh Research and Development.

[47] Ball, N., and Scurr, J. C. 2011. "Efficacy of Current and Novel Electromyographic Normalization Methods for Lower Limb High-speed Muscle Actions." European Journal of Sport Science 11 (6): 447-56.

[48] Konrad, P. 2005. The ABC of EMG. A Practical Introduction to Kinesiological Electromyography. Scottsdale, Arizona: Noraxon U.S.A. Inc.

[49] Winter, D. A. 2009. Biomechanics and Motor Control of Human Movement (4th ed.). Hoboken, NJ: Wiley.

[50] VencesBrito, A. 2012. "Kinesiological Characterization of Choku-tsuki Executed with Impact and Compared to the Execution without Impact." Revista de Artes Marciales Asiáticas 7 (1): 12-25. (in Portuguese)

[51] McGill, S. M., Chaimberg, J. D., Frost, D. M., and Fenwick, C. M. 2010. "Evidence of a Double Peak in Muscle Activation to Enhance Strike Speed and Force: An Example with Elite Mixed Martial Arts Fighters." Journal of Strength and Conditioning Research 24 (2): 348-57. 